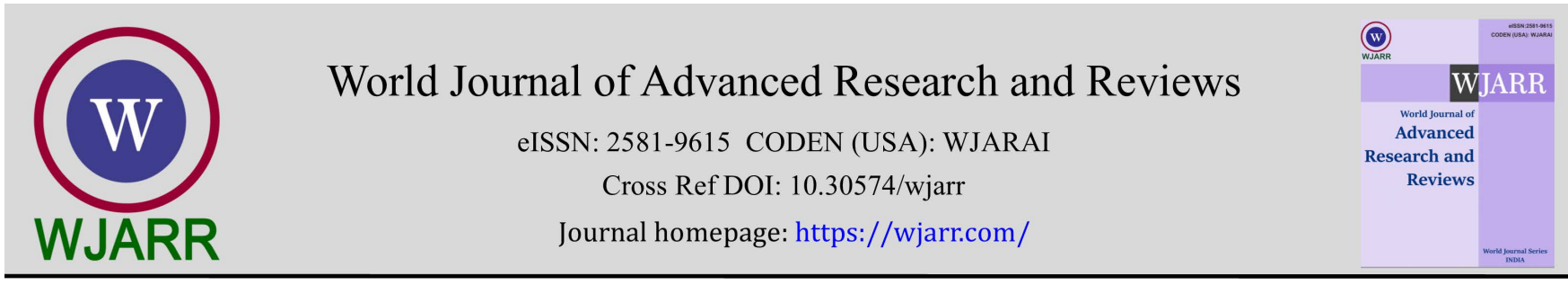

(RESEARCH ARTicle)

Check for updates

\title{
Psycho-social determinants and magnitude of public health problems of psoriasis in Bangladesh
}

\author{
Mohammad Samiul Huq *, Abu Hena Chowdhury, Towhida Noor and Saleheen Huq \\ ${ }^{1}$ Department of Dermatology, Square Hospital, Dhaka, Bangladesh. \\ ${ }^{2}$ Department of Dermatology, Bangabandhu Sheikh Mujib Medical University, Dhaka, Bangladesh. \\ ${ }^{3}$ Department of Dermatology, Dr Sirajul Islam Medical College Hospital, Dhaka. Bangladesh. \\ ${ }^{4}$ Department of Medicine, Peterborough City Hospital, England.
}

World Journal of Advanced Research and Reviews, 2021, 10(02), 108-118

Publication history: Received on 03 April 2021; revised on 07 May 2021; accepted on 10 May 2021

Article DOI: https://doi.org/10.30574/wjarr.2021.10.2.0207

\begin{abstract}
Background: Numerous studies have analyzed the influence of psoriasis on the quality of life and psychological health of patients. 0nly few studies have addressed the effect of the disease on individuals and cohabitants of psoriatic patients in developing countries, none in Bangladesh.
\end{abstract}

Objective: To assess the clinical severity, the physical and psychosocial disability and to analyze their interrelationship in psoriasis patients and cohabitants.

Methods: Hospital based cross-sectional study was conducted. The study included patients and cohabitants. The questionnaire was administered to the patient. Their quality of life was measured with the Psoriasis Disability index (PDI) and Family Dermatology Life Quality index (FDLQI), and their psychological state with Psoriasis life stress inventory (PLSI). The clinical severity by psoriasis area severity index (PASI) score. Appropriate test conducted using SPSS software.

Result: 225 patients (138 males, 87 female) were included in the study. The clinical PASI scores correlated significantly with the over all physical disability PDI $(<0.0001)$, stress incurred PLSI $(<0.0001)$, FDLQI $(<0.0001)$ and individual aspects of the PDI. The higher the PASI index, the higher the PDI, PLSI and FDLQI scores, which indicated greater impact on QOL. Among the physical and psychological factors analyzed, daily activity, employment, leisure and treatment were reported to be affected the most. Relative of female patients worries most.

Conclusion: Psoriasis markedly worsens the global well-being of patients and their cohabitants, who experienced an impairment of their quality of life and higher levels of anxiety and depression.

Keywords: Psychosocial; determinants; Quality of life; Health ; problems ; Psoriasis

\footnotetext{
${ }^{*}$ Corresponding author: Mohammad Samiul Huq

Department of Dermatology, Square Hospital, Dhaka, Bangladesh.

Copyright $(2021$ Author(s) retain the copyright of this article. This article is published under the terms of the Creative Commons Attribution Liscense 4.0.
} 


\section{Introduction}

Psoriasis is an immune-mediated disease (a disease with an unclear cause that is characterized by inflammation caused by dysfunction of the immune system) that causes inflammation in the body. There may be visible signs of the inflammation such as raised plaques (plaques may look different for different skin types) and scales on the skin [1].

Psoriasis is considered an incurable, long-term (chronic) inflammatory skin condition. It has a variable course, periodically improving and worsening. It is not unusual for psoriasis to spontaneously clear for years and stay in remission.

The self-esteem and quality of life of patients with psoriasis is often diminished because of the appearance of their skin. Recently, it has become clear that people with psoriasis are more likely to have diabetes, high blood lipids, cardiovascular disease, and a variety of other inflammatory diseases. This may reflect an inability to control inflammation.

Many patients with psoriasis think that they are stigmatized by the disease; the major aspects of the social burden of psoriasis include anticipation of rejection and feelings of shame and guilt. Other individuals may avoid touching people with psoriasis out of fear of contagious.

A variety of other aspects of the social burden of psoriasis have been identified by patients. Like effects on affected patients' interpersonal relationships. The impact of psoriasis on sexual well-being and the capacity for intimacy are primarily related to poor self-esteem and sexual self-image. Skin and sexuality are intrinsically linked, so avoidance of intimate situations among patients with psoriasis is common. Sexual distress is highest among patients with psoriatic lesions affecting their genitals, and women are typically more adversely affected than men.

The negative social impact of psoriasis is also apparent in the workplace (e.g., discrimination, difficulty finding employment or working outside the home, and financial hardships and/or lost income). Other occupational burdens related to psoriasis are increased patient absenteeism and reduced productivity. Nearly $60 \%$ of employed patients with severe psoriasis reported lost time from work in the previous year due to psoriasis; among those not working or retired, approximately one-third attributed this to psoriasis. In patients with psoriasis, health-related work productivity loss is predicted not by severity of disease but by health-related quality of life (QoL) [2].

Public health problem/agenda related to burden of psoriasis includes, prevalence, age of onset, direct costs, indirect costs health care utilization, employment/work burden, health related quality of life.

Like many other developing countries, In Bangladesh, burden of psoriasis definitely impact on health care system, as psoriasis yet to be included under NCD program of the country. Psoriasis patients are deprived from primary health facilities of the national health system.

The dual burden of non-communicable diseases (NCD) and infectious diseases upon the low and middle income counties are double burden. In fact, NCDs such cardiovascular diseases and diabetes are emerging and imposing now a new burden to those countries with limited resources and yet they are still struggling to meet the challenges of infectious diseases such as tuberculosis and HIV/AIDS [3].

Like other developing countries Bangladesh is also facing some challenges regarding unavailability of proper health care services. Study explore that there are many challenges in health care services such as inadequate infrastructure, population-bed ratio, doctor-nurse ratio, regional discrimination, low health expenditure, scarcity of drugs and high cost of rare drugs, high treatment cost, and unused of medical equipment in the public hospitals. Study also reveals that patient cannot access proper health care due to the illiteracy and poverty. Many of the cases, they are deceived by fraud to reach proper doctor and diagnosis services especially who comes from rural areas. Study also found that due to their poverty and unawareness, they are not able to get access better health care services from better places [4].

This study will show the multimodal effects of uncontrolled psoriasis on a patient's social framework. This might show a way that may help improve both the physical and social burden of the disease. In addition, this will determine the growing focus on assessments of the quality of healthcare, to determine the value for consumers, and how restructuring the healthcare system to pay-for performance or value-based purchasing could affect the care of psoriasis. As these new healthcare reimbursement practices are trending toward valuing measures of patient satisfaction, this study will also discuss how increased understanding of the social burden of psoriasis may lead to the provision of care in which treatment satisfaction is paramount. 


\section{Objective of study}

To assess the clinical severity, the physical and psychosocial disability and to analyze their interrelationship in psoriasis patients and cohabitants in developing countries like Bangladesh.

\section{Methodology}

\subsection{Sample}

The data collected from the dermatology outpatient clinic in Square Hospital Ltd, Dhaka, Bangladesh. Patients attending these clinics were a mixture of the rural and urban population from all over Bangladesh.

\subsection{Study design}

A cross-sectional prospective study. 225 patients with Psoriasis who attended Square Hospital OPD dermatology from November 2017 to June 2019. Abided hospital ethical regulations. Questionnaires given to the patients, after proper clinical examination.

\subsection{Inclusion and Exclusion Criteria}

The patients should have no other skin disease with visible patches.

The patients should be above 16 years of age.

The patients should be free from psychiatric illness.

The subjects were classified sex-wise, and age-wise as adolescents (14 to 17 years); adults (18 to 60 years) and old age (61 years and above).

\subsection{Tools for Measure}

\subsubsection{Clinical severity}

The extent of clinical severity of the disease was assessed by the Psoriasis Area Severity Index (PASI). Psoriasis Area and Severity Index (PASI) is the most widely used tool for the measurement of severity Psoriasis. PASI combines the assessment of the severity of lesions and the area affected into a single score in the range 0 (no disease) to 72 (maximal disease).

\subsubsection{Quality of life assessment}

Patients completed a multidimensional QOL assessment comprising the psoriasis disability Index (PDI), The Psoriasis Life Stress Inventory (PLSI) and Family Dermatological Quality of Life Index (FDLQI). All the questionnaires are in English and been interpreted in Bengali by the interviewing Doctor.

\section{Psoriasis disability}

The original PDI (1990 version) [5] questionnaire was suitably modified to suit the local population. The resulting score ranged from 0 to 45. Each question score 3. The higher the score, the more the quality of life is impaired. The PDI can also be expressed as a percentage of maximum possible score of 45 . As in the original questionnaire, all the questions related to events that had taken place in the preceding 4 weeks.

\section{Psoriasis life stress}

We assessed the stress incurred by the patient using a modified version of the psoriasis life stress inventory (PLSI), Scores on this scale ranged from 0 to 45 . The scores can also be expressed as percentage scores [6].

Family dermatological quality of life index

The Family Dermatology Life Quality Index [7] is a questionnaire designed for adult (more than 16 years of age) family members or partners of patients with any skin disease. It is self-explanatory and can be simply handed to the patient's family member/partner who is asked to fill it in without the need for detailed explanation. It is usually completed in two to three minutes. 
The FDLQI total score is calculated by summing the score of each question resulting in a maximum of 30 and a minimum of 0 . The higher the score, the more quality of life is impaired. The FDLQI can also be expressed as a percentage of the maximum possible score of 30 .

\subsection{Statistical analysis}

Statistical analysis was conducted using SPSS for Windows (Release 7.5.1) statistical software.

Non-parametric variables were subjected to Pearson's Chi-square analysis; parametric variables were analyzed using Student's t-test and analysis of variance (ANOVA). Spearman's correlation analysis was carried out for select variables.

\section{Results}

The study group consisted of 225 consecutive consenting patients with psoriasis attending the OPD of dermatology department of Square Hospital Ltd, Dhaka.

Overall, 225 patients (16-69 years old, 138 men, 87 women) who had interviewed with the English Version of PDI, PLSI and FDLQI, completed all the questions. The mean age of the respondents was $37.94(\mathrm{SD}=13.8)$ years. Scores of the PDI, PLSI, FDLQI and PASI ranged from 7 to 36 (mean \pm SD, $21.28 \pm 6.13$ ), 7 to 38 (mean \pm SD, 23.6 \pm 7.94 ), 6 to 24 (mean $\pm \mathrm{SD}, 11.56 \pm 4.4$ ) and 4.33 to 60.66 (Mean \pm SD, 17.22 \pm 11.71 ), respectively. The characteristics of the respondents are given in table 1 .

Using Student T test, Disease duration has no significant correlation with PID. $(\mathrm{p}=0.06)$ Also, reliability coefficient in unmarried patients and married cases was not significant in the PDI. There was no significant difference in clinical severity or total QOL scores between the two genders.

The mean score of PASI in our cases was 17.22 (out of the maximum possible score of 72), mean score of PDI was 21.28 (out of the maximum possible score of 45), Mean score of PLSI 23.6 (out of Maximum possible score 45, Mean score of FDLQI 11.56 (out of maximum possible score of 30)

Table 1 characteristics of the respondents

\begin{tabular}{|l|c|c|}
\hline Patient Characteristics & Value & STDEV \\
\hline Total number of respondent's & 225 & \\
\hline Number of Male & 138 & \\
\hline Number of Female & 87 & \\
\hline Mean age in years & 37.94 & 13.8 \\
\hline Male & 39.42 & 13.81 \\
\hline Female & 35.73 & 13.73 \\
\hline Mean duration of Disease & 16.17 & 11.05 \\
\hline Male & 16.55 & 11.81 \\
\hline Female & 15.6 & 9.97 \\
\hline Mean age at onset & 21.98 & 8.02 \\
\hline Male & 23.08 & 7.4 \\
\hline Female & 20.33 & 8.65 \\
\hline Mean PASI Score & 17.22 & 11.71 \\
\hline Male & 17.61 & 11.96 \\
\hline Female & 16.63 & 11.52 \\
\hline Mean PDI Score & 21.28 & 6.13 \\
\hline
\end{tabular}




\begin{tabular}{|l|c|c|} 
Male & 21.84 & 6.59 \\
\hline Female & 20.43 & 5.3 \\
\hline Mean PLSI Score & 23.6 & 7.94 \\
\hline Male & 23.73 & 8.34 \\
\hline Female & 23.4 & 7.42 \\
\hline Mean FDLQI Score & 11.56 & 4.4 \\
\hline Male & 10.35 & 3.23 \\
\hline Female & 13.36 & 5.29 \\
\hline
\end{tabular}

There was no significant difference in clinical severity or physical disability between the two groups based on the patients present age. Patient's age of onset of psoriasis were not significant with the QOL scores. Using Pearson's correlation coefficient we observed highly significant correlation of the PASI score with the total PDI, PLSI and FDLQ. Table 2

Table 2 Correlation matrix showing the relationship between PASI, PDI, PLSI and FDLQI Pearson's correlation ( $\mathrm{r}$ )

\begin{tabular}{|l|l|l|l|l|}
\hline & PASI & PDI & PLSI & FDLQI \\
\hline PDI & $\mathrm{R}=0.647(\mathrm{p}=0.00001)$ & & $\mathrm{R}=0.7018(\mathrm{p}=0.0001)$ & \\
\hline PLSI & $\mathrm{R}=0.573(\mathrm{p}=0.00001)$ & & & $\mathrm{R}=0.391(\mathrm{p}=0.0005)$ \\
\hline FDLQI & $\mathrm{R}=0.636(\mathrm{p}=0.00001)$ & $\mathrm{R}=0.523(0.00001)$ & & \\
\hline
\end{tabular}

Correlation between clinical severity score and various aspects of physical disability. We observed significant correlation of the PASI score with all subdivisions of PDI table 3.

Table 3 Pearson's correlation coefficient for relation between total PASI scores and subdivisions of PDI.

\begin{tabular}{|l|l|l|}
\hline \multirow{2}{*}{ PDI Subdivisions } & \multicolumn{2}{|c|}{ PASI } \\
\cline { 2 - 3 } & R value & P Value \\
\hline Daily activities & 0.635 & 0.00001 \\
\hline Employment & 0.645 & 0.00001 \\
\hline Personal relations & 0.462 & $3 \mathrm{E}-05$ (highly significant) \\
\hline Leisure & 0.520 & 0.00001 \\
\hline Treatment & 0.333 & 0.003 \\
\hline
\end{tabular}




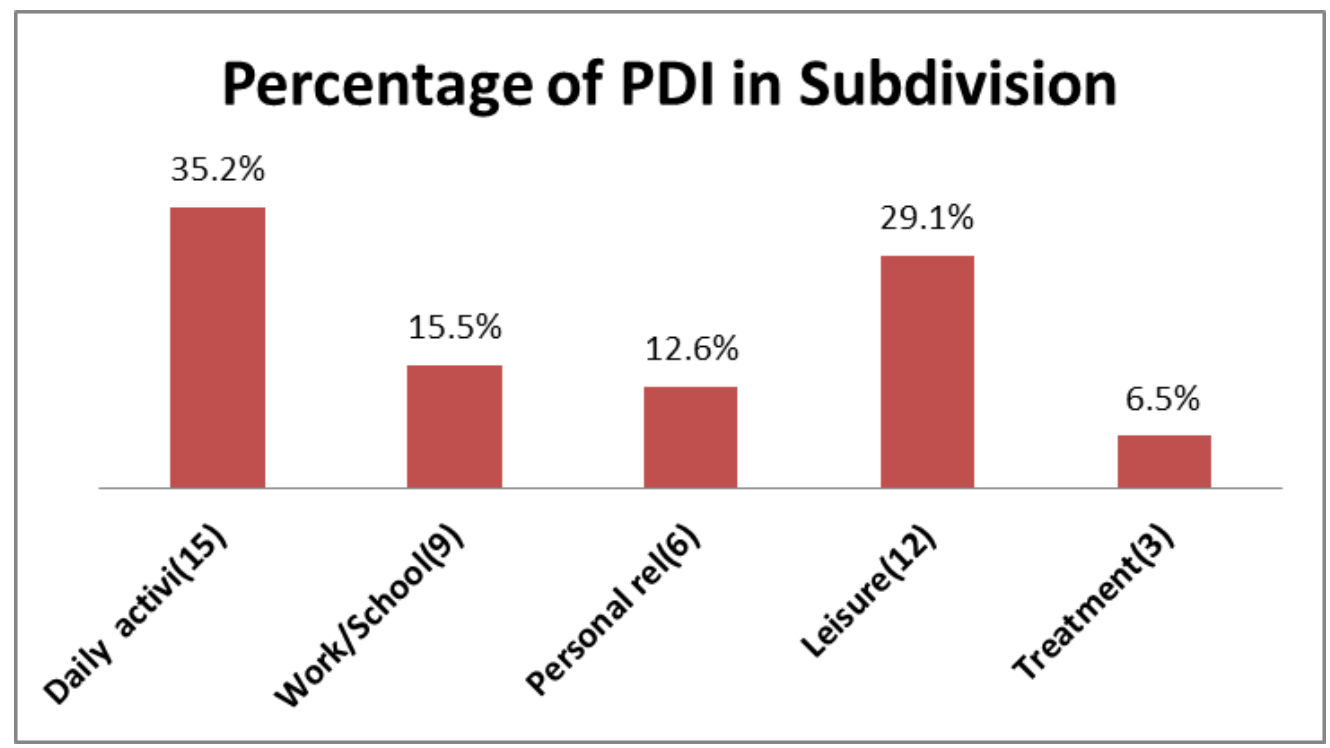

Figure 1 Percentage of PDI in subdivision

In Figure 1 shows daily activities and in leisure time they were affected most as shown in Psoriasis debilities index of Psoriasis patients.

The patients were divided into 2 groups based on their PASI scores; group I, PASI < 11; group II, PASI > 11. The mean of the PDI and its subdivisions and the PLSI and FDLQI in each of the clinical severity groups were compared table 4. We found the total PDI and all its subdivisions.

Table 4 Difference in the mean scores of total PDI, PLSI and FDLQI between two clinical severity groups, PASI $<11$ and PASI $>11$

\begin{tabular}{|l|l|l|l|}
\hline QOL indices & PASI $<\mathbf{1 1} \mathbf{~ N = 1 0 8}$ & PASI $>\mathbf{1 1} \mathbf{~ N = 1 1 7}$ & P value \\
\hline Daily activities & $5.86 \pm 1.89$ & $9.38 \pm 1.84$ & 0.00001 \\
\hline Employment related & $2 \pm 0.71$ & $3.82 \pm 1.18$ & 0.00001 \\
\hline Personal Relationships & $2.13 \pm 0.96$ & $3.35 \pm 0.96$ & 0.00001 \\
\hline Leisure & $5.30 \pm 1.54$ & $7.61 \pm 1.67$ & 0.00001 \\
\hline Treatment related & $1.13 \pm 0.54$ & $1.58 \pm 0.63$ & 0.0007 \\
\hline Total PDI & $16.44 \pm 4.57$ & $25.76 \pm 4.60$ & 0.00001 \\
\hline Total PLSI & $18.97 \pm 7.59$ & $27.87 \pm 5.53$ & 0.00001 \\
\hline Total FDLQI & $9.91 \pm 2.45$ & $13.07 \pm 5.22$ & 0.0007 \\
\hline
\end{tabular}

On the basis of the PLSI scores (percentage scores), we divided our patients into 2 groups: group I - less stress reactive group, with a PLSI score of < 10; group II - high stress group, with a PLSI score of $>10$. On comparing the 2 groups, we observed that patients classified as more reactive to stress had higher clinical severity score (PASI, $\mathrm{P}=0.008$ ) and higher physical disability (PDI, $\mathrm{P}<0.00001$ ) and also the cohabitants are affected more (FDLQI, $\mathrm{P}=0.0 .007)$. We also observed that group II had higher mean scores in all aspects of PDI. Table 5 
Table 5 Statistical difference in means of total PDI, total PASI and FDLQI among the two stress groups based on PLSI scores

\begin{tabular}{|l|l|l|l|}
\hline QOL indices & PLSI $<\mathbf{1 0} \mathbf{~ N ~} \mathbf{2 7}$ & PLSI $>\mathbf{~ 1 0 ~ N ~ = ~ 1 9 8 ~}$ & P Value \\
\hline Mean PASI & $8.62 \pm 1.20$ & $18.39 \pm 12.02$ & 0.008 \\
\hline Mean PDI & $11.77 \pm 3.07$ & $22.59 \pm 5.72$ & 0.00001 \\
\hline Mean FDLQI & $8.22 \pm 0.66$ & $12.01 \pm 4.50$ & 0.007 \\
\hline Daily activities & $4.33 \pm 1.22$ & $8.15 \pm 2.35$ & 0.00001 \\
\hline Employment related & $1.55 \pm 0.52$ & $3.13 \pm 1.31$ & 0.0003 \\
\hline Personal relationship & $1.44 \pm 0.72$ & $2.95 \pm 1.07$ & $5.6 \mathrm{E}-05$ Highly significant \\
\hline Leisure & $3.55 \pm 1.01$ & $6.90 \pm 1.72$ & 0.00001 \\
\hline Treatment related & $0.88 \pm 0.60$ & $1.43 \pm 0.61$ & 0.006 \\
\hline
\end{tabular}

The overall pattern of response, percentage of patients ticking each response in the FDLQI questionnaires are given in Figure 2.

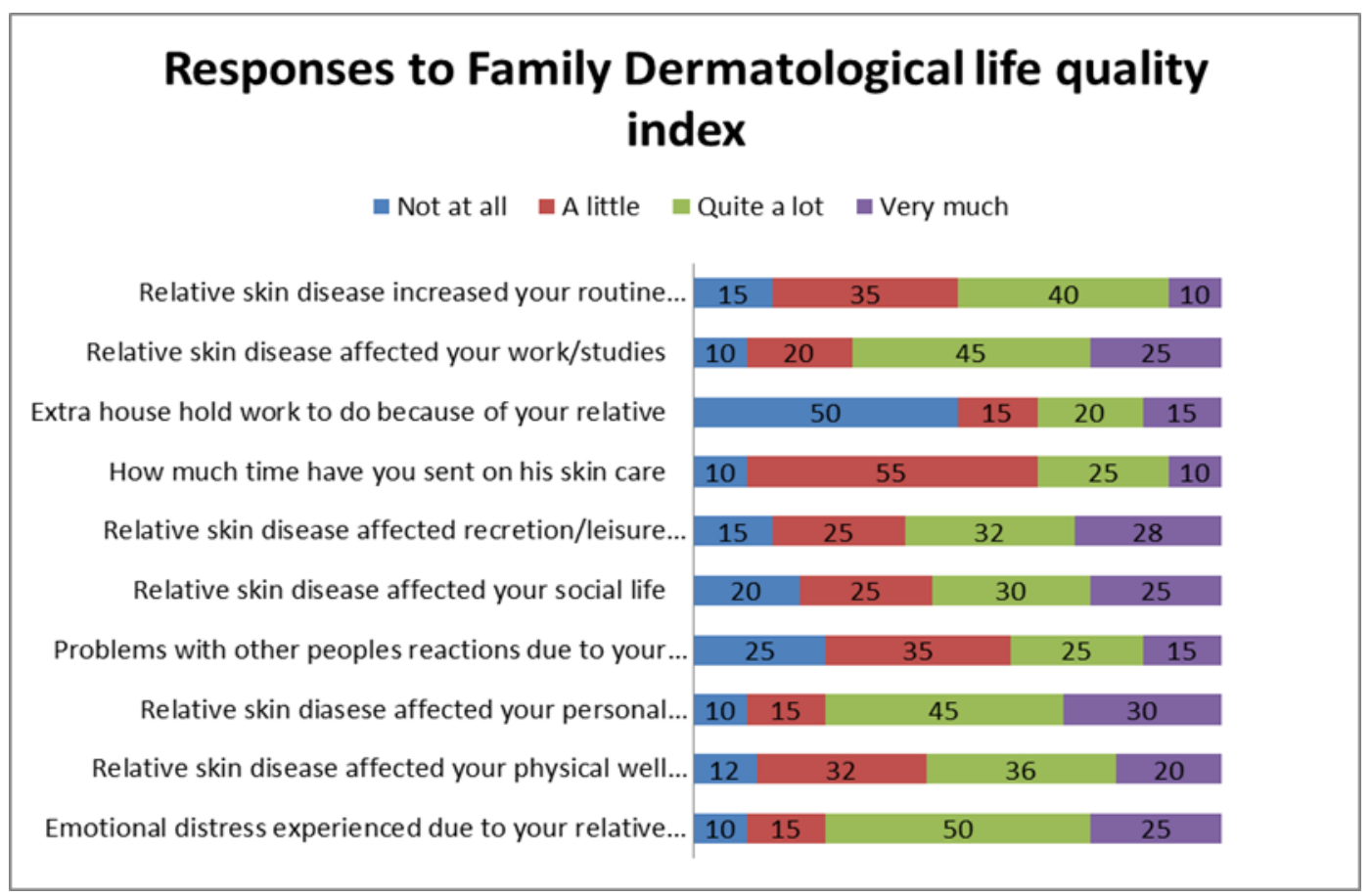

Figure 2 Responses to family dermatological life quality index

The presence of psoriasis impaired the quality of life of the cohabitants. FDLQI scores of cohabitants were significantly associated with the PDI and PLSI scores of the patients $(\mathrm{r}=0.523 ; \mathrm{P}<.00001)$ and $(\mathrm{r}=0.391 ; \mathrm{P}<0.0005)$. Anxiety and depression levels did not differ between patients and cohabitants, 50 per cent stated that the treatment of their relative or partner resulted in them having to spend extra time on housework; Most of them described psychological pressures including anxiety, being upset and being worried about the patient's future; Social disruption due to lack of social confidence either because of embarrassment or because of the time required for care duties; $30 \%$ described limitations to holiday plans, sport and leisure activities and evenings out; $70 \%$ described limitations on their daily activities such as shopping, work and time spent with other family members; Many felt that their close relationships had deteriorated. 


\section{Discussion}

Psoriasis has a tremendous impact on patient's lives, affecting them physically, psychologically and socially, as well it affect the family members $[8,9]$. It has public health problem. A study showed that the impact of psoriasis on HRQOL is similar to that of other major medical disease such as cancer, arthritis, hypertension, heart disease, diabetes and depression. When asked, patients who have more than one of the major medical problems almost always preferred to have their psoriasis cured over other major medical problems [10].

Our study showed that physicians may need to improve relations with their patients and should re-evaluate their management of psoriasis. Better sympathy and communication between the psoriasis patients and their physicians may help to improve clinical outcomes in psoriasis. This research may serve as a stimulus to better understand this communication and to use more aggressive treatment options.

Psoriasis represents a life long burden for the affected patients. There is a general consensus among research studies that objective clinical severity alone is insufficient as an assessment of the burden of disease. It is the inner world of the patient that clinicians need to assess. One study reported that only 39\% of patients who had psoriasis with clinically relevant distress were identified correctly by dermatologists [11]. And when physicians did identify patients as clinically distressed (anxiety or depression), further action to address such difficulties through referral to appropriate specialists was taken in only one-third of cases despite the potential effects of distress on adherence to treatment and the effectiveness of treatment [12,13]. Therefore, QoL measures are being assigned increasing importance in the evaluation of health care outcomes [14]. Indian clinicians and researchers have recently started to give importance to this aspect of psoriasis. Our study will be discussed to give a better perspective of what disability psoriasis patients experience both physically and psychosocially

The psycho-social and occupational impact of psoriasis is as important as traditional physical parameters such as PASI. [15] Although the PASI has its disadvantages, to date, it is the most adequate instrument available to evaluate clinical severity in plaque type psoriasis.[16] PDI, PLSI and FDLQI are popular tools used to assess QOL in psoriasis all over the world.[17]

The clinical severity measurement correlated significantly with the overall physical disability (PDI), individual aspects of the PDI, measurement of stress incurred (PLSI) and measurements of anxiety, depression and quality of life (FDLQI). Our findings were in concordance with those of other investigators like Finlay,[18] Aschroft,[19] and Gelfand [20] who also found moderate correlation between PASI scores and PDI scores. Yang et al [21] and Fortune et al,[22] however, did not find any significant correlation between PASI scores and PDI scores. On closer examination of Fortune's study, we noted that most of his patients had low clinical severity scores (mean PASI $=8.8$ ) as compared to our study (mean PASI = 17.22 \pm 11.71 ). Koo et al. [23] had demonstrated earlier that PDI is a more reliable instrument for people with severe psoriasis, who have more physical disability and limitations related to life styles. Hence the extensive clinical involvement in most of our patients causing physical disability and imposing limitations on their life styles, explains the highly significant correlation between PASI and PDI in this study. This is in contrast to Fortune's [22] patients, who had milder disease so as not to interfere with their life styles. Recently, the psychometric properties of PDI have been analyzed, leading to the conclusion that PDI is not well targeted to measuring disease impact among patients with low Level of disability.[24]

We also observed significant correlation ( $\mathrm{r}=0.647 ; \mathrm{P}<.00001)$ of the PASI scores with all subdivisions of PDI, study had found the PASI scores to significantly correlate with all aspects of PDI. Subject with a more severe clinical disease if they are treated in state owned hospital would actually face lesser financial burden than the patient with clinically milder disease, who would have been treated as an outpatient and would have had to buy his own medicines.

In our study, we observed significant correlation ( $\mathrm{r}=0.573 ; \mathrm{P}<0.00001)$ between the overall PASI scores and the stress incurred by the patient (PLSI). Despite a thorough search of literature, we came across only two studies [21] which attempted to correlate the PASI and PLSI scores. This study by Fortune [22] found no correlation between clinical severity and psoriasis life stress inventory. We assume, again, his patients with mild clinical disease probably had lower stress levels to be reflected in the overall PLSI scores. Earlier, Gupta [5] found correlation between PLSI scores and global patient self rating of clinical severity of the disease.

In our study, a PASI score of more than 11 delineated a subgroup of patients with higher overall physical disability and higher stress rating. Aspects of daily activities, employment, and personal relationships were the most affected in this subgroup with higher clinical severity. Thus, a cut off point for PASI (PASI > 11), which was earlier reported $\{25]$ as an 
indication for starting systemic therapy, also served to delineate a subgroup of people with more or less physical disability and psychological stress.

A PLSI score of more than 22.5\% (equivalent to the standardized cut off of 10), labeled as 'high stress reactors,' also served to identify a subgroup of patients with greater overall physical disability scores and higher clinical severity scores. Gupta,[5] and later Fortune, [22] had classified their patients on the basis of PLSI score into 2 groups: Those patients who reacted significantly to stress associated with having psoriasis (score of $>10$ ) and those patients who were not significantly affected by psoriasis-related stress (score of $<10$ ). For comparative purposes, we also divided our patients into 2 groups: high stress reactors (score of $>10$ ) and low stress reactors (score of $<10$ ). We found that patients who were classified as more reactive to stress (PLSI score $>10$ ) associated with psoriasis had no relation to the earlier age of onset of the disease, a higher clinical severity score $(\mathrm{P}=0.0001)$, and more overall physical disability $(\mathrm{P}=0.000)$ in all areas of everyday life. The aspects of psoriasis disability which bore significant difference in the 2 groups were daily activities $(\mathrm{P}<0.00001)$, employment or alternative questions $(\mathrm{P}<0.0003)$, leisure $(\mathrm{P}<0.00001)$, highly significant with personal relationship and treatment-related activities $(\mathrm{P}<0.006)$. Fortune [22] also found that his high stress reactive patients of psoriasis perceived themselves as disabled in all areas of everyday life as assessed by PDI. Gupta[26] observed that a PLSI score of more than 10 delineated patients with greater overall psoriasis severity $(\mathrm{P}=0.008)$, more cosmetically disfiguring psoriasis, greater number of flare-ups of psoriasis, and greater severity of pruritus.

Analysis of the PDI questionnaire on the basis of percentage of response for each aspect of disability [Figure 1] showed that questions related to daily activities, and leisure scored the highest, and the questions related to difficulties in personal relationships and employment, treatment related scored the lowest. Whereas findings in Finlay's [27] study group with a mean score of $38.2 \%$. In his study, the mean sub-scores of daily activities and treatment section were greater than those of the other 3 sections, the questions relating to personal relationships scoring the lowest. More than $50 \%$ of patients in his study with 'a lot' or 'very much' to 2 questions relating to clothes; questions relating to baths, sports, and home messiness. That the questions related to sports bore contrasting results with our study can be explained by the difference in the importance given to sports activities in the late adulthood between the two cultural settings. Less than $30 \%$ of patients in Finlay's [27] study, as in our study, responded with 'a lot' or 'very much' to questions relating to career, problems with friends or partners, and smoking and/or alcohol. PDI has been validated against short form health survey, or SF-36 [28,29]; sickness impact profile, or SIP [18]; and Salford psoriasis index, or SPI [30]. All these investigators found overall PDI and all aspects of PDI to be affected in their psoriasis patients.

Among the psycho-social measures investigated, psoriasis sufferers were found most likely to feel self conscious, be disturbed / inconvenienced by the shedding of the skin, live in a constant fear of relapse, and avoid social situations. Stressful events in which psoriasis patients were discriminated against by others were least reported. The stressful events resulting from cosmetic disfigurement seem to be more prevalent in our patients. Questions relating to stressful events in which the psoriasis patients were discriminated against by others - people treating them as contagious, insensitive remarks by adults/children, barber appearing reluctant to cut hair - all were endorsed by a lower percentage of patients. The pattern of answering by our patients bore resemblance with the responses of psoriasis patients in the US [5]. This clearly shows that the pattern of problems of psoriasis patients is universal. The stress from cosmetic disfigurement and stress from coping with physical aspects of psoriasis seem to take the highest toll as indicated by both our patients' responses and Gupta's [5] patients' responses to PLSI questionnaire.

Ginsburg, [31] in her study of stigmatization found that being older at onset of psoriasis protects people against anticipating rejection, feeling sensitive to opinion of others, feeling of guilt and shame, and secretiveness. Our study is not concordance with these finding. There was no relation with age of onset, or gender activities. Gupta and Gupta [26] also showed that men report more occupational impairment but Koo et al, [23] found that women reported more severe disease than man. Surprisingly the duration on of the disease had no significant impact on the mean PASI or mean PDI scores. Similar findings had been reported by Fortune et al. [22] This suggests that the range of impact of psoriasis is not simply reducible to the chronicity of the disease.

\section{Conclusion}

Psoriasis markedly worsens the global well-being of patients and their cohabitants, who experienced an impairment of their quality of life and higher levels of anxiety and depression. This study will create awareness among community and health care system in developing countries where psoriasis is yet to be included in national non communicable disease (NCD) control program. 


\section{Compliance with ethical standards}

\section{Acknowledgments}

We are grateful to all participants and the team at the Department of Dermatology, Square Hospital ltd, for supporting the research and Mr Reasad Azim to input the data in computer for analysis.

\section{Statement of informed consent}

Informed consent was obtained from all individual participants included in the study.

\section{References}

[1] Singh JA, Guyatt G; American College of Rheumatology/National Psoriasis Foundation Guideline for the Treatment of Psoriatic Arthritis; Journal of Psoriasis and Psoriatic arthritis; Vol 4;Issue 1;2018; November 30.; 31-58

[2] Alexa BK, Christine J, Stefan N, et al. The psychosocial burden of psoriasis; Am J clin Dermatol. 2005; 6(6): 36392.

[3] Mahmood S, Islam R; Shifting from infectious disease to non communicable disease: A double burden of diseases in Bangladesh; Journal of Public Health and Epidemiology: Vol.5[11];November 2013,pp.424-434,

[4] Islam A, Biswas T. Health System in Bangladesh: Challenges and Opportunities; American Journal of Health Research. 2014; 2(6): 366.

[5] Lewis VJ, Finlay AY. Two decades experience of the psoriasis disability index. Dermatology. 2005; 210: 261-8.

[6] Gupta MA, Gupta AK; Psoriasis life stress inventory; A preliminary index of psoriasis related stress, Acta Derm Venereol. 1995; 75: 240-3.

[7] Martinez-Garcia E, Arias-Santiago S. Quality of life in person living with psoriasis patients; J Am Acad Dermasol. 14 May 2014.

[8] Monali JB, Amit Kulkarni; Quality of life in patients with psoriasis; health and quality of life outcomes. 2006; 4: 35.

[9] Basra MKA, Finlay; The family impact of skin disease; the greater patient concept; BJD. May 2007; 156(5): 929937.

[10] Fried RG, Friedman S, Paradis C et al. Trivial or terrible? The psychosocial impact of psoriasis. Int. J. Dermatol. 1995; 34: 101-105.

[11] Richards HL, Fortune DG. Detection of psychological distress in patient with psoriasis; low consensus between dermatologist and patients, BJD. 151: 1227-1233.

[12] Renzi C, Picardi A, Abeni D. Association of dissatisfaction with care and psychiatric morbidity with poor treatment compliance. Arch Dermasol. 2002; 138: 337-42.

[13] Richards HL, Fortune DG, O’Sullivan TM; Patients with psoriasis and their compliance with medication. J Am Acad Dermatol. 1999; 41: 581-3.

[14] Finlay AY; The outcomes movement and new measure of psoriasis; J Am Acad Dermatol. 1997; $36: 502-3$.

[15] Kirby B, Richards HL, Woo P, Hindle E, Main CJ, Griffiths CE. Physical and psychological measures are necessary to assess overall psoriasis severity. Journal of the American Academy of Dermatology. 2001; 45(1): 72-76.

[16] Schmitt J, Wozel G. The psoriasis area and severity index is the adequate criterion to define severity in chronic plaque type psoriasis. Dermatology. 2005; 210: 194-9.

[17] Lewis VJ, Finlay AY; Two decades experience of the psoriasis disability index. Dermatology. 2005; 210: 261-8.

[18] Finlay AY, Khan GK, Luscombe DK, Salek MS. Validation of sickness impact profile and psoriasis disability index in psoriasis. Br J Dermatol. 1990; 123: 751-6.

[19] Ashcroft DM, Li Wan Po A, Williams HC, Griffiths CE. Quality of life measures in psoriasis: A critical appraisal of their quality. J Clin Pharm Therapeutic. 1998; 23: 391-8. 
[20] Gelfand JM, Feldman SR, Stern RS, Thomas J, Rolstad T, Margolis DJ. Determinants of quality of life in patients with psoriasis: A study from the US population. J Am Acad Dermatol. 2004; 51: 704-8.

[21] Yang Y, Koh D, Khoo L, Nyunt, SZ, Nq V, Goh CL. The psoriasis disability index in Chinese patients: Contribution of clinical and psychological variables. Int J Dermatol. 2005; 44: 925-9.

[22] Fortune DG, Main CJ, O'Sullivan TM, Griffiths CE. Quality of life in patients with psoriasis: The contribution of clinical variables and psoriasis specific stress. Br J Dermatol. 1997; 137: 755-60.

[23] Koo J. Population-based epidemiologic study of psoriasis with emphasis on quality of life assessment. Dermatol Clin. 1996; 14: 485-96.

[24] Nijsten T, Whalley D, Gelfand J, Margolis D, McKenna SP, Stern RS. The psychometric properties of the Psoriasis Disability Index in United States patients. J Invest Dermatol. 2005; 125: 665-72.

[25] Griffiths. WHO Model prescribing Information-Drugs used in Skin Diseases. Clin Exp Dermatol. 1999; $24: 50$.

[26] Gupta MA, Gupta AK. Age and gender differences in the impact of psoriasis on quality of life. Int J Dermatol. 1995; 34: 700-3.

[27] Finlay AY, Coles EC ; The effect of severe psoriasis on the quality of life of 369 patients. ; Br J Dermatol; 1995; 132: 236-44.

[28] Ware JE Jr, Sherbourne CD. The MOS 36-item short form Health survey (SF-36). Med Care. 1997; 30: 473-81

[29] O' Neill P, Kelly P. Postal questionnaire study of disability in the community associated with psoriasis. Br Med J. 1996; 313: 919-21

[30] Kirby B, Richards HL, Woo P, Griffiths CE; Physical and Psychological measures are necessary to assess overall psoriasis severity. J Am Acad Dermatol. 2001; 45: 72-6.

[31] Ginsburg, I. H., \& Link B. G. (1993). Psychosocial consequences of rejection and stigma feelings in psoriasis patients. International journal of dermatology. 1993; 32(8): 587-591 